\title{
An Improved SRS Noise Estimation Algorithm Based on Interference Cancellation
}

\author{
Kewen Liu ${ }^{1, a}$, Weiwei $\operatorname{Tian}^{1, b}$, Mingyue Wang ${ }^{1, c}$, Xianglin Chen ${ }^{1, d}$ and Jihua \\ Gong $^{1, \mathrm{e}}$ \\ ${ }^{1}$ Mobile Communications Lab, Wuhan University of Technology, Wuhan,Hubei 430068, China \\ aliukewen@whut.edu.cn, btianweiwei@163.com, cwangmingyue@126.com, \\ dchenxianglin@126.com, ${ }^{\mathrm{e}}$ gongjihua@126.com
}

\section{Keywords: LTE;SRS;Interference Cancellation;Noise Estimation}

\begin{abstract}
This thesis studies SRS noise estimation method with multi-users in the Long Term Evolution(LTE), and proposes a Discrete Fourier Transform (DFT) time-domain filtering algorithm based on interference cancellation. By calculating signal power in the system protecting band, decision threshold of time domain filtering can be denoted. Therefore, one can reduce the impact of noise on the useful signal within the window, and also obtain a more accurate noise estimation. Simulation results show that either in the case of single-user or multi-user, the proposed algorithm can get good performance. At the same time, the algorithm can accurately reflect the impact in neighborhood interference on each band channel quality, so as to achieve the frequency domain resource selective scheduling effectively.
\end{abstract}

\section{Introduction}

As a new generation of mobile communication technology,LTE standard adops carrier frequency division multiple access (SC-FDMA) for uplink, orthogonal frequency division multiple access (OFDMA) for downlink transmission [1]. OFDM technology has high spectral efficiency, bandwidth scalability, anti-multipath fading, frequency domain scheduling and adaptive advantage . However, when the number of independently modulated subcarriers consistent with the use, OFDM symbols have a very high peak-to-average ratio (PAPR), which increases the cost and power consumption of the transmitter amplifier and is unsuitable to be used for terminal , so the uplink channel using the SC-FDMA which has a single carrier property. Through the base station scheduler, UE side implement multi-user multiplexing in the frequency and time domain on PUSCH. And base station side check the channel quality of each user for resource scheduling [2].An important UL reference signal,th Sounding Reference Signal(SRS) is defined in support of frequency dependent scheduling,power control and UL synchronization maintenance.

By estimating the signal to interference noise ratio(SINR), SRS can reflect the channel quality information of each user on each RB. At the same time offset can be estimated without sending Physical Uplink Shared Channel (PUSCH) and Physical Uplink Control Channel(PUCCH), thus the eNode achieve resource scheduling and time compensation. Covering the whole system bandwidth, SRS can get entire channel bandwidth channel quality information on each RB. Then choosing the channel quality band as a Shared channel frequency band, and next time slot it can be used to strengthen the power control. How to detect the SINR on each RB is the SRS research priorities, and the key is to estimate multiuser noise and interference on each RB. In [3],some SNR estimation algorithms are compared in AWGN in detail. [4] proposes a method of channel estimation based on IFFT in the time domain to calculate the noise estimate value.However, at large SNR, the signal energy leakage significantly, leading to smaller signal power estimation, and SNR estimation error is large. [5] proposes an algorithm by comparing MSE of channel coefficients to select a window function. Even though it can get a better performance, in practice, this decision conditions is not easy to achieve. In [6] noise value can be estimated better, but one cyclic shift is reserved exclusively,lending to reduce the number of multiplexed users. 
To solve how to accurately estimate the SINR for each user on each RB problem in a multi-user code division multiplexing case, according to the structural characteristics of the SRS transmission mode and LTE uplink, this paper presents an improved SRS channel quality estimation algorithm based on interference cancellation. The algorithm not only improves the SINR estimation accuracy in single-user situations, but also be able to accurately estimate the multi-user environment SINR each $\mathrm{RB}$, and provides an accurate judgment basis for uplink frequency selective scheduling.

\section{SRS Transmission Model}

As to FDD-LTE frame structure,a radio frame is $10 \mathrm{~ms}$ long and consists of 10 subframe of length $1 \mathrm{~ms}$.A subframe is defined as two consecutive slots of $0.5 \mathrm{~ms}$,each made of seven SC-FDMA symbols(normal cyclic prefix configuration) and demodulation reference signal (DMRS)in the middle. When the subframe is configured for SRS transmission,the last symbol is reserved for the SRS[1].The SRS subframe number is determined by the cell level and UE level period configuration.

The FDD-LTE frame structure is depicted in Fig.1.SRS transmission of the configured sub-frame is always located in the last SC-FDMA symbol. Demodulation reference signal (DMRS) and sounding reference signal (SRS) located at different SC-FDMA symbols. DMRS is associated with the transmission of PUSCH and PUCCH. Assigned to the SRS symbol does not allow for PUSCH transmission.However ,SRS has nothing to do with PUSCH and PUCCH transmission.

Multiple UEs can be multiplexed in a combination of FDM and CDM through high-level configuration and scheduling. SRS uses CAZAC sequence as an uplink reference signal (DMRS / SRS) sequence.

SRS is definded according to

$$
r_{u, v}^{(\alpha)}(n)=e^{j \alpha n} \bar{r}_{u, v}(n), \quad 0 \leq n<M_{\mathrm{sc}}^{\mathrm{RS}}-1
$$

where $M_{\mathrm{sc}}^{\mathrm{RS}}=m N_{s c}^{R B}$ is the length of the reference signal sequence and $1 \leq m \leq N_{R B}^{\max , U L}$. Multiple reference signal sequences are defined from a single base sequence through different values of $\alpha$.Base sequences $\bar{r}_{u, v}(n)$ are divided into groups, where $u \in\{0,1 \ldots, 29\}$ is the group number and $v$ is the base sequence number within the group[1]. $\alpha$ is the base sequence of cyclic shift and the same base sequences of different shift will form a different reference signal sequences. The cyclic shift $\alpha$ of the sounding reference signal is given as

$$
\alpha=\frac{2 \pi n_{\mathrm{cs}}}{8}
$$

where $n_{\mathrm{cs}}$ is configured for each UE by higher layers and $n_{\mathrm{cs}}=0,1,2,3,4,5,6,7$.

Multi-user SRS signal can be transmissioned through code division and frequency division multiplexing. The transmission strcture of CDM-based two-user is shown in Fig.2.

The revieved SRS can be expressed as:

$$
Y=H_{1} X_{1}+H_{2} X_{2}+N
$$

where $X_{1}, X_{2}$ are the transmitter frequency domain signal of UE1 and UE2. $X_{i}(n)=e^{j \alpha_{i} n} \bar{r}_{u, v}(n)$, $0 \leq n<M_{\mathrm{sc}}^{\mathrm{RS}}, \alpha_{i}=2 \pi \frac{\mathrm{n}_{\mathrm{SRS}}^{\mathrm{cs}}}{8} ; H_{1}, H_{2}$ are the channel estimation coefficient of UE1 and UE2; and $N$ is the noise signal.

\section{Noise Estimation Algorithm}

\section{Noise estimation based on DFT}

First we get MMSE (minimum mean square estimation) of channel frequency response $H_{L S}(k)$ 


$$
H_{L S}(k)=Y(k) X^{*}(k)=H(k)+V(k)(1 \leq k \leq N)
$$

Where $\mathrm{N}$ for the length of SRS signal; $H(k)$ for the actual channel coefficients; $V(k)$ for noise portions.After IDFT, we get the time domain impulse response $h_{L S}(n)$

$$
h_{L S}(n)=\operatorname{IFFT}\left\{H_{L S}(k)\right\}=h(n)+v(n)(1 \leq n \leq N)
$$

Suppose $L$ response to the impact of the channel length. When $n>N, h_{L S}(n)=0$. Generally, Since the channel impulse response length is less than the length of the cyclic prefix,it is considered that the channel impulse response beyond the cp part consists of the noise. Thus the noise power can be expressed as

$$
\hat{\sigma}_{D F T}^{2}=\frac{1}{N-N_{c p}} \sum_{n=N_{c p}}^{N}\left|h_{L S}(n)\right|^{2}
$$

\section{Noise estimation based on interference elimination}

For example ,two user code division multiplexing (CDM). SRS signal received by the base station can be expressed as: $Y=H_{1} X_{1}+H_{2} X_{2}+N$. Then the entire frequency band noise and interference can be expressed as

$$
N=Y-H_{1} X_{1}-H_{2} X_{2}
$$

where $H_{1}$ and $H_{2}$ are the Channel coefficient of user1 and user2, which can be estimated by LS; $X_{1}, \quad X_{2}$ Can be generated locally by the base station.So the noise and interference can be calculated by 6 .

\section{Improved Algorithm}

Due to the first estimate of the channel coefficient error of conventional noise estimation method based on interference cancellation is large, so it is far from the actual deviation of the $\mathrm{N}$ and the resulting performance is poor. The traditional DFT noise estimation method is just to calculate the average noise power on the entire band, estimate the SINR unable to show the differences on each RB clearly. Therefore we propose an improved algorithm under interference cancellation method. In order to obtain accurate channel of each user estimated coefficients, we will fill the LS estimate of the coefficient of zero transform to time domain, then add window to filter out the useful signal. But there are still parts of the noise within the window, which have a greater impact on the resulting $\mathrm{N}$. Due to the LTE system left protection band distribution at both ends, we can think only noise, we can calculate this part of the noise power as decision threshold of noise filtering within the window.

The specific implementation of the algorithm flow chart is shown in figure 3, the implementation steps as follows:

1.After removing the $\mathrm{CP}, 7.5 \mathrm{KHz}$ frequency offset compensation and FFT frequency domain ,the received data is recorded as $Y$, which is the superposition of all user data in the frequency domain. Calculating the signal power on the guard band without effective data transmission as initially noise $\hat{\sigma}_{I D F T}^{2}$ for all users of the cell this TTI(transmission time interval).

2.Then do subcarrier demapping and get SRS data corresponding to the subcarriers of length $M_{\mathrm{sc}}^{\mathrm{RS}}$.

3.The sequence after demapping do conjugate multiplication with ZC base sequence $\bar{r}_{u, v}(n)$, then zero padding IDFT changes to time domain (zero-padded to prevent edge effects).If CDM users exist in this band, there will be a corresponding time-domain impulse signal by $\mathrm{n}_{\mathrm{SRS}}^{\mathrm{cs}}$ to distinguish.To separate multiuser in time domain, windowing is added in time domain. If there is no time offset effect, impulse signal should be located in $\frac{\left(8-\mathrm{n}_{\mathrm{SRS}}^{\mathrm{cs}}\right) \times N_{I D F T}}{8}$, window length $\frac{N_{I D F T}}{8}$. To counter 
the time offset, the window function on both sides is extended to $\frac{C P / 2 \times N_{I D F T}}{N_{F F T}}$. Then remove the time domain cyclic shift.

4. Although the time-domain filtering removes noise outside the window, the noise is still left within the window.Seting the threshold, the noise can be removed within the window. $\mathrm{K}_{\mathrm{th}}$ is set for the selected threshold ,before DFT we only retain the samples whose power greater than $K_{t h} \cdot \hat{\sigma}_{I D F T}^{2}(a)$ (sideband initial noise). For example, in the user window, i represents the sample point.If $\left|\hat{h}_{u}^{S R S}(i, a)\right|^{2} \leq K_{t h} \cdot$ srs_noise $(a)$, then $\hat{h}_{u}^{\text {SRS }}(i, a)=0$. Otherwise keep its data.(Notice that this kind of noise removal can't remove the noise hidden in the symbol, so frequency domain noise removal is very important )

5. Do DFT transformed to the frequency domain, and remove excess zeros.The length of the original signal is restored and we can obtain channel estimation coefficients $H_{1} 、 H_{2} \ldots H_{n}$.

6. Using the channel estimation value to calculate signal power:

$$
\begin{aligned}
& E_{P R B, u}(a)=\frac{1}{6}\left(\sum_{i \in P R B}\left|\tilde{H}_{u, i, a}^{S R S}\right|^{2}\right)-\hat{\sigma}_{D F T}^{2}(u, a) \\
& \hat{\sigma}_{D F T}^{2}(u, a, P R B) \approx \frac{L_{t h}^{u}}{M_{D F T}} \text { srs_noise }
\end{aligned}
$$

Where $L_{t h}^{u}$ for the reserved valid points in time domain. The above calculation is the signal power of a receiving antenna, for the case of multiple antennas.The $E_{P R B, u}(a)$ obtained in each antenna are added and averaged.

7. In order to calculate the noise power, using a similar method SIC,when detecting data for each UE, we can put the other UE's data as interference to eliminate . After the useful signal are removed, the resulting signal is considered as interference and noise. Specifically, if there are two UE code division multiplexing.For UE1, signal after interference cancellation is noise and UE2 data. $Y^{\prime}=Y-H_{1} X_{1}$. When detecting UE2, UE2 useful signal is eliminated and noise signal is obtained. $N=Y^{\prime}-H_{2} X_{2}=Y-H_{1} X_{1}-H_{2} X_{2}$, where $N$ for the signal on frequency domain. $N$ is used to calculate noise power in each PRB(the addition averaging on Multiple antennas):

$$
\hat{\sigma}_{P R B}^{2}(a)=\frac{1}{6}\left(\sum_{i \in P R B}\left|N_{i, a}\right|^{2}\right)
$$

8. SINR and the average SINR is calculated for each UE on each RB

$$
\begin{aligned}
& s n r_{-} P R B_{-} d B=10 \log \frac{E_{P R B, u}}{\hat{\sigma}_{P R B}^{2}} \\
& s n r_{-} d B=10 \log \left(\frac{1}{n_{-} P R B} \sum_{i=1}^{n_{-} P R B} \frac{E_{P R B, u}}{\hat{\sigma}_{P R B}^{2}}\right)
\end{aligned}
$$

\section{Simulation}

\section{Simulation scenario and parameter Settings}

The simulation environment as shown in table 1 : 
Table 1 Simulation Parameters

\begin{tabular}{|l|l|}
\hline System Bandwidth(MHz) & 20 \\
\hline Number of antennas & 2 \\
\hline Channel & EPA5 \\
\hline Number of SRS users & $1 \sim 2$ \\
\hline SNR range(dB) & {$[-10,+20]$} \\
\hline SRS bandwidth(RB) & 80 \\
\hline Simulation times & 500 \\
\hline
\end{tabular}

\section{Simulation result}

Measure: the average SINR and MSE

The average SINR: average of 500 measurements of SNR

$$
\text { aver_snr_dB }=\frac{1}{N_{T}} \sum_{i=1}^{N_{T}} s n r_{-} d B_{i}
$$

Mean square error (MSE) is defined:

$$
M S E=\frac{1}{N_{T}} \sum_{i=1}^{N_{T}}\left(s n r_{-} d B_{i}-S N R_{\text {ideal }}\right)^{2}
$$

Where $s n r_{-} d B_{i}$ for the i-th estimated average SNR, $S N R_{\text {ideal }}$ for a real value, $N_{T}$ for the number of simulations.

The following are the algorithm performance simulation verification of single UE and 2UE scenarios.

case A: single UE

In EPA5,the time-domain filtering algorithm based on the interference cancellation is compared performance with [4] and [7]. Fig. 4 for MSE performance comparison.

Fig. 4 shows that, in the case of a single UE, the proposed elimination of interference improved algorithm outperforms traditional algorithms based IFFT. The new algorithm and Qiu's are both relatively accurate, but the MSE of new proposed algorithm is always less than Qiu's algorithm with higher stability of the estimated SINR.

case B : 2UE CDM

2 UE transmit code division multiplexed together and pass the EPA5 channel. The time-domain filtering algorithm based on the interference cancellation is compared performance with [7]. Fig.5 for MSE performance comparison.

Trough different ncs $(0,2)$ code-division multiplexing,the SRS bandwidth of two UE are both 80RB. The figure only shows the performance curve of one UE, another is similar situation. From Fig. 5 shows that when the channel environment is poor (SNR $<10 \mathrm{db}$ ), the proposed algorithm is superior to the Qiu's, which can better reflect the channel quality information. When the channel environment is better, the impact of noise on SINR measurement is reduced and the performance of the two algorithms are equivalent..

case C: $2 U E$ plus cell interference

2 UE transmit code division multiplexed together and pass the EPA5 channel.At the same time, adjacent CellInterference is added to the cell SRS and the influence of interference to the algorithm performance is measured.From the Fig.6,the blue part is the average SINR performance curve of new algorithm $10 \mathrm{~dB}$. Where the interference on the upper half band,SINR $=0 \mathrm{~dB}$. The red part is the average SINR performance curve of new algorithm 20dB. Where the interference on the upper half band,SINR=10dB.

Fig. 6 shows the performance SINR estimation curve on each RB after adding SRS neighbor cell interference at $\mathrm{snr}=10 \mathrm{~dB}$ and $20 \mathrm{~dB}$. The simulation result shows that the proposed algorithm fully embodies the differerence between adding interference on the low frequency band and no interference on the high frequency band of SINR. Ater adding adjacent CellInterference on the low 
frequcency band,whether there is interference or not on the band,the real SINR can be estimated accurately.

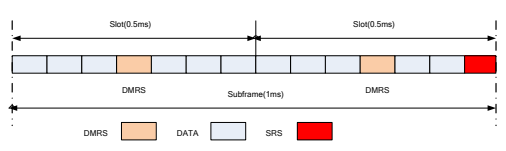

Fig.1 FDD-LTE frame structure

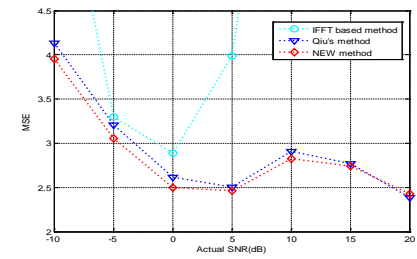

Fig.4 Single UE EPA5

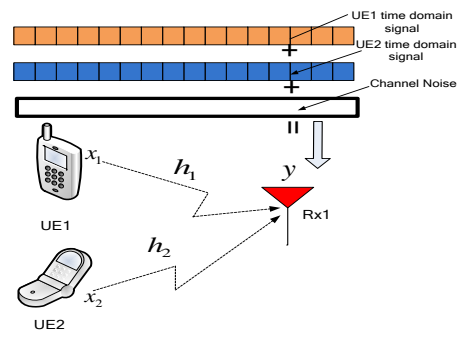

Fig.2. SRS transmission model

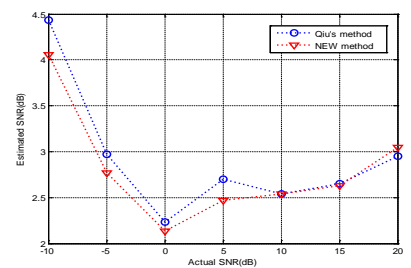

Fig.5 2UE(CDM)

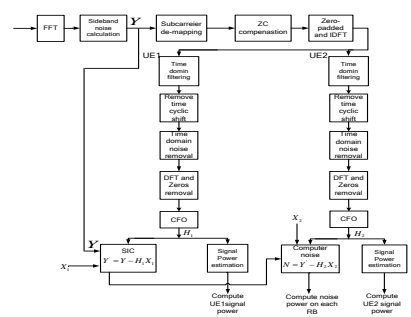

Fig.3 Receiver flow chart

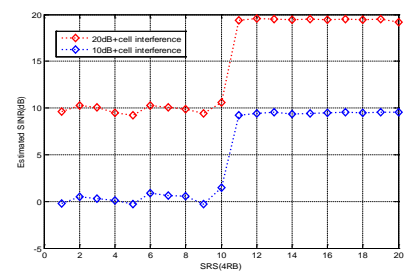

Fig.6 2UE(CDM) Adjacent CellInterference

\section{Conclusions}

Based on the features and functions of the SRS signal, a novel SINR estimation algorithm is proposed. The algorithm is based on the idea of interference cancellation, combined with DFT algorithm in the time domain filtering. Simulation results show that the new proposed algorithm not only in single-user and multi-user situations measured SINR performance accurately, but also in the adjacent cell interference can detect the channel quality information in each band accurately, which is benefit for base stations to achieve frequency domain selective scheduling.

\section{Acknowledgements}

This work was supported by Natural Science Foundation of Hubei Province(NO.2013CFB349).

\section{References}

[1] 3GPP TS 36.211 V11.0.0,2012-09, Technical Specification Group Radio Access Network; Evolved Universal Terrestrial Radio Access (E-UTRA)[s]; Physical Channels and Modulation (Release 11).

[2] Safa H, Tohme K. LTE uplink scheduling algorithms: Performance and challenges[C]Telecommunications (ICT), 2012 19th International Conference on. IEEE, 2012: 1-6.

[3] D. Pauluzzi and N. Beaulieu, "A comparison of SNR estimation techniques for the AWGN channel”, IEEE Trans. Commun., vol. 48, no. 10, pp. 1681-1691, 2000.

[4] Fei J, Guangliang R, Zhe Z. A new noise variance and post detection SNR estimation method for MIMO OFDM systems[C]Communication Technology, 2008. ICCT 2008. 11th IEEE International Conference on. IEEE, 2008: 179-182.

[5] Tian H, Yang L, Li S. SNR estimation based on sounding reference signal in LTE uplink[C] Signal Processing, Communication and Computing (ICSPCC), 2013 IEEE International Conference on. IEEE, 2013: 1-5.

[6] Bertrand P. Channel gain estimation from sounding reference signal in LTE[C] Vehicular Technology Conference (VTC Spring), 2011 IEEE 73rd. IEEE, 2011: 1-5.

[7] Qiu Shi, LI Pingan, MaTing. The research of noise estimation algorithm on LTE uplink sounding reference signal [J]. 2013. 